\title{
IMPLEMENTATION OF LRU ALGORITHM FOR LOCATION UPDATE IN WIRELESS M2M COMMUNICATION
}

\author{
Samruddhi C. Thawkar ${ }^{1}$ \\ ${ }^{1}$ Computer Science and Engineering Department, G.H. Raisoni College of Engineering, Nagpur, India
}

\begin{abstract}
Wireless M2M communication is about enabling the flow of data between machine and machine and ultimately between machine and people. For communication it is necessary to trace the Machine-Type-Communication (MTC) device successfully so novel energy and memory efficient location update scheme for wireless M2M communication is studied which is more efficient for a MTC device to perform location updates only when new nodes are added into the network to avoid call dropping ratio. Flexible and distributed Wireless M2M communication is robust and rapidly deployable/reconfigurable, so they are highly appealing for a lot critical applications, like deep space communication, disaster relief, battlefield communication, outdoor mining, car that react in real time to prevent accidents etc. This paper presents different paging algorithms that are studied on the basis of certain parameters such as endto-end delay, compression rate, data transmission energy and call-mobility-ratio. Least Recent Used (LRU) algorithm is implemented for optimally pruning the nodes from the network for efficient location update which is considered as the best paging algorithm.
\end{abstract}

Keywords: Location Update, LRU paging, Memory Management, etc ****.

\section{INTRODUCTION}

Machine-to-machine (M2M) communication is about enabling the flow of data between machines and machines and ultimately machines and users. M2M communications represents a future where billions of everyday objects and the surrounding environment are connected and managed through a variety of devices, communications networks, and cloudbased servers[4].

An energy-and-memory efficient location update scheme for wireless M2M communications is studied. In which, the Machine-Type-Communication (MTC) server track the location of each M.T.C. device but does not know the mobility patterns of M.T.C. devices in advance. It is widely assumed that a variety of wireless access networks will exist in the near future, the proposed location update scheme is designed to be independent of the wireless access networks. The total number of M.T.C. devices is expected to exceed the human population, it is required to optimize the location update cost for each M.T.C. device.

A novel algorithm based on random walks over tree is introduced to prune the parsing tree optimally for location update cost, the size of a parsing tree grows with time but the memory size of a M.T.C. device is limited and may not be as large as a smart phone. So, an algorithm is proposed to reduce the sum of memory and location cost[3].

Paging is one of the most important memory management schemes by which a computer can store and retrieve data from secondary storage for use in memory. A LRU paging will be implemented for optimal pruning the tree to reduce memory cost . To avoid call dropping and managing memory efficiently we will implement LRU paging with location update techniques.

The rest of the paper is organized as follows. Related works are covered in Section II. In Section III, we have studied various location update techniques and their benefits and drawbacks. In Section IV, we have discussed page replacement algorithms for best performance of memory management. Section V propose a system location update scheme based on LRU paging to optimally prune the parsing tree by replacing the nodes. Section VI describes future scope of work. Our conclusions are included in Section VII.

\section{EXISTING LOCATION UPDATE TECHNIQUES}

The location update procedure allows a mobile device to inform the cellular network, whenever it moves from one location area to the next. Mobiles are responsible for detecting location area.

\subsection{Profile Based Strategy}

Personal communication system (PCS) is characterized by high user density and high mobility so registration will incur a large amount of radio link signaling traffic which results a large number of handovers. This increases the messaging and consumes a large amount of scarce bandwidth and require additional signaling system 7(SS7). The main objective of Profile Based Strategy is to reduce the signaling traffic on the radio link by increasing the efficiency of network. In this 
technique, system maintains a sequential list of the most likely places where each user is located. The list is ranked from most to least likely place where a user is located. When a user moves between location areas in this list, location update is not required. The list may be provided by the user or may be based on each user's past calling history. The users are divided into three classes depending upon the predictability of their daily routine.

Deterministic Users: This includes the users which follow a rigid daily routine that the system knows. The user never needs to register, and the location area therefore be as small as possible. If the user happens to move out of the area (a rare event), then the call cannot be completed

Quasi-Deterministic Users: These users are found in work during a certain time and at home during another time interval. The time intervals vary slightly from day to day. There may be alternate routes on the way to and from work. During lunch, they might be found at different restaurants. These users display a certain likelihood of being in a particular place, but in actuality, they might not be there.

Random Users: This includes users whose location is almost unpredictable. Past knowledge of their location cannot predict their future location. The profile-based strategy of this paper is not applicable to these users.

The main limitation of this strategy is that the mobility pattern is known in advance, and if mobility is known in advance then location update is not required.

\subsection{Group Registration Strategy}

In mobile networks the location of a mobile needs to be traced successfully for efficient call delivery. As mobile user changes location area, a registration request is sent to home location register (HLR) to update user profile with new location area which results a high volume of signaling traffic. The main task Group Registration (G.R.) strategy is to efficiently reduces the location registration cost by updating location changes to the Home Location Register (H.L.R.) for multiple mobile terminals (M.T.s) in a single location update request message. The I.D.s of the M.T.s newly moving into an Location Area (L.A.) are buffered and received by H.L.R. for location update in the route response message of the next incoming call to any M.T. in the L.A[4].

The main factor that determines the performance of Group Registration Strategy is when and how to send the request to the HLR for location update. The request is sent to HLR upon arrival of next incoming or outgoing call for any mobile terminal in the location area.

This strategy reduces location tracking signaling cost and call delivery latency in mobile networks and has achieved a cost reduction over wide range of Call-mobility-ratio (CMR).This strategy is based on concept of location area as it is already in mobile system and does not require system to collect mobility and calling results for mobile terminals[6].

The main drawback of this strategy is that it does not benefit users with extremely high CMR as the total cost per arrival is negligible.

\subsection{Adaptive Location Management Strategy Combining Distance Based}

A new adaptive scheme in which an optimal distance-based update threshold is selected not only as a function of the callto-mobility ratio, but also as a transitional directivity index, a new parameter is introduced to give some measures of the mobile's travelling patterns[8]. Its advantage becomes even more significant when the optimal threshold is not obtained due to certain restrictions imposed by the network during times of high system loading.

\subsection{Parsing Tree Strategy for Location Update}

In parsing tree strategy energy and memory efficient location update scheme is proposed. In comparison with periodically registering to Machine-Type-Communication (MTC) server it is efficient for a MTC device to perform location updates only when new nodes are added into the parsing tree. The M.T.C. device maintains a parsing tree and a queue, which is initially empty. A parsing tree is a well-known data structure in the field of source coding.

Memory management in parsing tree for MTC devices is done by novel approach which optimally prune i.e. removal of excess nodes, the parsing tree. It is desired to prune the tree optimally as the total number of MTC devices is expected to exceed the human population.

This technique overcomes the limitation of above two strategies i.e. the mobility pattern is not known in advance and is independent of wireless access networks. Due to the optimal pruning of tree there is less chance of signaling traffic. Removal of excess nodes from queue minimize the value of memory cost and energy cost for a MTC device.

\section{PAGE REPLACEMENT ALGORITHMS}

During a program execution, some data are required to be loaded into the primary memory. In the memory management, based on paging mechanism, the required data are entered to the memory as pages contents. When a required page is not found in memory, a page fault event is occurred. In this case, the required page must be loaded into memory. Some page replacement algorithms are studied: 


\subsection{First- In-First-Out}

In this algorithm, page that has entered to the memory before all other pages, will removed from the memory before them. The idea behind this is that, the first entered page has enough chance to be used and this chance must be given to another page. When a page needs to be replaced, the page at the front of queue is selected and is removed. This algorithm is easy to understand and use but practical application is very poor[10].

The first disadvantages is if a page is used frequently in several time periods, it will be identified as the last or oldest page, and may be selected to be moved out from the memory, while there is a considerable probability for urgent need to it then the selection will be inefficient and the removed page must be reloaded into memory immediately.

Another disadvantage for this algorithm is that it increases the memory frames designated for a process and can cause lower page fault ratio and the performance of this algorithm degrade as the number of pages increase.

\subsection{Not Recently Used}

The strategy included in N.R.U., is that a page without any change, during its residence in primary memory, can be known as a desirable page to be removed from the memory. This algorithm uses two status bits named Reference bit (R) and Modification bit (M). These bits are contained in each page table entry, and the algorithm initializes them with zero, for all pages. When a page is referred to or its contents change, $\mathrm{R}$ or $\mathrm{M}$ will be set, respectively. These bits must be updated for each page referring, it is vital that they be set by the hardware[11].

When there is a need to replace a page with a new one, first, it is attempted to find a page without any reference $(\mathrm{R}=0)$.If no such page was found, a page with $\mathrm{R}=1$, preferably with $\mathrm{M}=0$ (without change) will be selected. The reason for such this selection is that removing pages with change $(M=1)$, impose a secondary memory rewriting overhead. With different states for $\mathrm{R}$ and $\mathrm{M}$ four major groups of pages are:

Class 0: Not Referenced, Not modified $(\mathrm{R}=0, \mathrm{M}=0)$

Class 1: Not Referenced, Modified ( $R=0, M=1)$

Class 2: Referenced, Not Modified $(\mathrm{R}=1, \mathrm{M}=0)$

Class 3: Referenced, Modified ( $\mathrm{R}=1, \mathrm{M}=1)$

The groups with lowest number include the pages with more priority to exit the memory and the later groups have minimum priority.

\subsection{Least Recently Used}

In this algorithm the pages are widely used in the last few iterations, will be used heavily again, in the next few ones. And, in contrast, the pages that have not been used for long times, will probably remain unused afterward. So, this algorithm selects a page that its last usage is before all other resident pages.

This algorithm has a suitable performance. The best way to implement LRU is using stack. In this case, each invoked page is inserted on top of stack. So the pages that have been used more recently are put on the stack top and the least used pages at the bottom of the stack.

Among all the page replacement algorithms LRU is the one with best performance, the LRU algorithm uses for each page, the number of times that page has been referred recently[10].

\subsection{Second Chance Algorithm}

Second chance algorithm is the modified first in first out that prevent from removing pages that are widely used. As same in Not Recently Used algorithm, the second chance algorithm

Selects a page to be moved out on the basis of reference bit(R).If it is zero, the intended node is considered as useless and is replaced with new one. But if $R=1$, it is cleared that node will be moved to the end of queues. The search will continue till $\mathrm{R}=0$ otherwise it will work same as first in first out[11].

\section{COMPARATIVE ANALYSIS OF ABOVE TECHNIQUES}

We have studied three paging algorithms location update in wireless M2M communication. From TABLE I, First in first out paging algorithm is not much efficient than other two algorithms as energy efficiency is low as compared to others, also time required to replace the node is also average. The second algorithm is Not recently used (NRU) which has given better performance than previous algorithm. The third algorithm has given best performance but it haven't considered compression rate which is the most promising factor of paging. Hence, the fourth technique i.e. Least Recent Used algorithm can be best to study further as compared to other two algorithms.

Table I. comparative analysis of location updates techniques

\begin{tabular}{|l|l|l|l|l|}
\hline $\begin{array}{l}\text { Sr } \\
\text { N } \\
\text { o }\end{array}$ & $\begin{array}{l}\text { Paging } \\
\text { Algorithm }\end{array}$ & Delay & $\begin{array}{l}\text { Energy } \\
\text { Efficienc } \\
\text { y }\end{array}$ & $\begin{array}{l}\text { Compressio } \\
\text { n Rate }\end{array}$ \\
\hline 1. & $\begin{array}{l}\text { First in first } \\
\text { out }\end{array}$ & Medium & $30 \%$ & $\begin{array}{l}\text { Not } \\
\text { Considered }\end{array}$ \\
\hline 2. & $\begin{array}{l}\text { Not Recently } \\
\text { Used }\end{array}$ & High & $30-50 \%$ & $\begin{array}{l}\text { Not } \\
\text { Considered }\end{array}$ \\
\hline 3. & $\begin{array}{l}\text { Second } \\
\text { Chance } \\
\text { Algorithm }\end{array}$ & Medium & $50 \%$ & $\begin{array}{l}\text { Not } \\
\text { Considered }\end{array}$ \\
\hline
\end{tabular}




\section{PROPOSED TECHNIQUE}

In this section, the proposed methodology will be implemented to get an efficient location update in terms of delay, energy efficiency and compression rate. This section includes definitions, flow of technique, network model and a LRU Paging Algorithm.

\subsection{Definitions}

Delay: It is the time required to replace the old node by the new active node. Average delay is calculated by averaging the delay of all the nodes replaced by new nodes.

Compression Rate: Reducing the number of nodes used in representing a piece of information, by eliminating the the least recent used node. Compression ratio is the most important factor for memory management.

Energy Efficiency: It is the energy required by the nodes to replace least used nodes with widely used nodes. The energy of a network is obtained by averaging over all energy of nodes in the network.

\subsection{Flow of Technique}

This section explains the overall flow of implementation of location update based on LRU technique as shown in fig 1 .

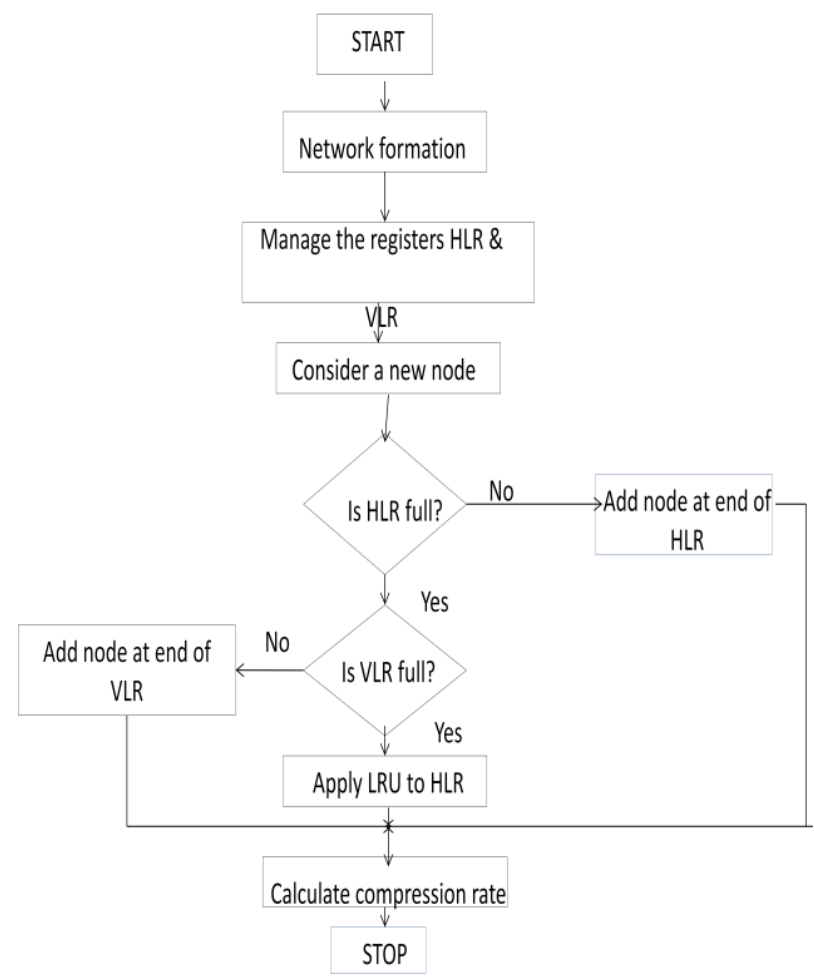

Fig 1 Flow of Location Update Technique in M2M Communication

\subsection{Network Model}

We have considered a dynamic network which is divided into two registers Home Location Register(HLR) and Visitor Location Register(VLR). Total nodes considered here are 30. Every node is set with parameters.. The packet size is $1000 \mathrm{kB}$ and packet interval is $0.0001 \mathrm{sec}$. Every node is registered in HLR and roaming node is in VLR. For successful call delivery, paging algorithm is implemented to trace the respective node efficiently.

\subsection{Least Recent Used Paging Algorithm}

As per considered in above network, there are total 30 nodes according to their location they are located in the respective registers. To replace old and useless node LRU paging will be implemented with a new an active node for efficient call delivery to avoid call dropping.

Home Location Register: It is a local stack which stores the local nodes registered in the network located at their home location.

Visitor Location Register: It is a visitor stack where roaming nodes are located which are out of their respective location.

These nodes when move out of their home register they have to register again in the VLR for which extra charges is applicable i.e roaming charges as they are out of their home location registers.

The idea behind this algorithm is that, devices used in last few instructions will probably be used again in next few ones and in contrast, the device not been used for long times will probably remain unused.

With the help of this algorithm, it replaces node with least recent used node and it does not remove the node permanently from tree which overcomes the call dropping. L.R.U. has a suitable performance. For memory management, L.R.U. will be implemented on both the memory management registers i.e H.L.R. and Visitor Location Register (V.L.R.) which results successful tracing of location and will results prominent call delivery.

Every time home location register and visitor location register are updated. If we want to update location all the old least recent used nodes is replaced by active nodes so as to improve the performance of the network.

Every time $\mathrm{N}$ is selected as new node, it executes the following Algorithm 1.

Algorithm 1: LRU Paging Algorithm

1. Network is divided into two register HLR \& VLR

2. New node $\mathbf{N}$ is considered

3. N checks whether HLR is full or not 

4. If HLR is full
5. $N \cdot \cdots-$
6. Fig 2. Multi-hop Relay Technique in MANETs
7. $\mathrm{N}$ executes step1
8. else
9. $\mathrm{N}$ is added at end of HLR
10. Else if
11. $\mathbf{N}$ is added at end of VLR
12. End if

Step 1: Implementation of LRU in HLR

1. New node $\mathrm{N}$ randomly search location in HLR

2. If HLR is full then

3. least recent used node for longer period is replaced by $\mathrm{N}$

4. HLR is updated

5. Compression rate is calculated

6. End if

This Least Recent Used(LRU) algorithm will optimize delay performance, energy efficiency and compression rate in highly dynamic network of M2M communication. The implementation process of this algorithm is in progress.

\section{IMPLEMENTATION}

\subsection{Formation of Network}

First step of proposed plan has been implement that is formation of network. In this step, 30 nodes are considered. Network formation consists of nodes formation, position formation, communication between nodes and connection. Nodes are numbered from 1 to 30 . Node 0 is considered as old node which is to be replaced. According to their location nodes are moving from home to visitor registers and vice versa. It is simple communication method, percentage of call drop is very high here.

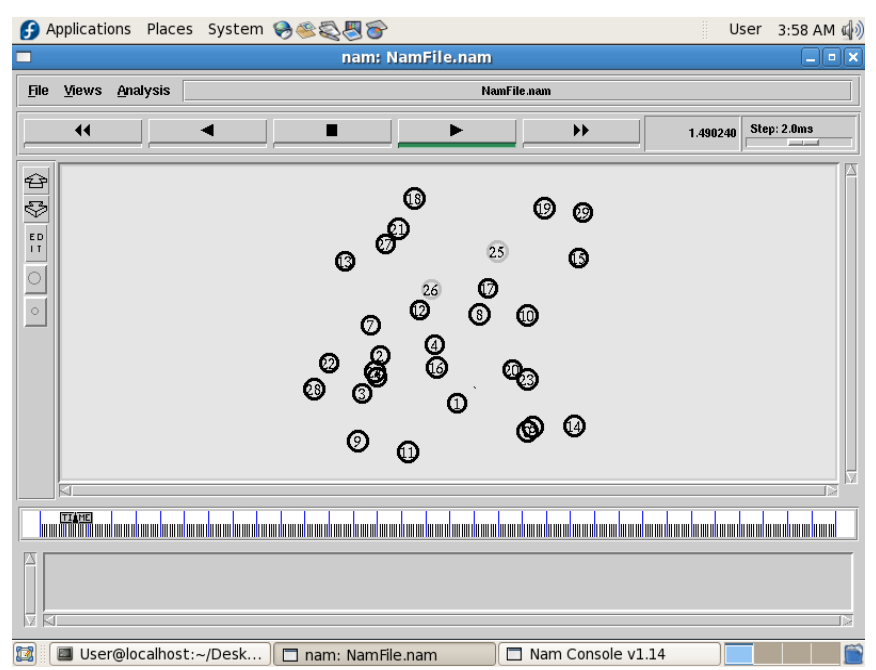

\section{CONCLUSIONS}

The location update techniques and three paging algorithms are explained, have been compared and analyzed on the basis of three parameters that are delay, energy efficiency and compression rate. To overcome the drawbacks of these three algorithms a least recent used (LRU) algorithm is suggested to improve location update in Wireless M2M communication. The proposed scheme has added a concept that simultaneously minimizes delay as well as improves energy efficiency and compression rate in highly dynamic network. It is believed that the proposed scheme will work efficiently and gives optimal result.

\section{ACKNOWLEDGMENTS}

This is my final year $\mathrm{ME}$ research project in wireless communication and computing under the guidance of Ms.V.A.Gulhane. The work completed up to now is presented in this paper. The work is still under construction. The results will be presented in the future. The research work in this paper was supported by G. H. Raisoni College of engineering, Nagpur.

\section{REFERENCES}

[1] G. Wu, S. Talwar, K. Johnsson, N. Himayat, and K. D. Johnson, "M2M: from mobile to embedded internet," IEEE Commun., Apr. 2011.

[2] A. Roy, A. Misra, and S. K. Das, "Location update vs. paging tradeoff in cellular networks: an approach based on vector quantization," IEEE, Dec. 2007.

[3] F. Akyildiz, J. S. M. Ho, and Y.-B. Lin, "Movementbased location update and selective paging for PCS networks,".,Aug. 1996.

[4] Z. Mao and C. Douligeris, "Group registration with local anchor for location tracking in mobile networks," IEEE Trans. May 2006.

[5] E. Cayirci and I. F. Akyildiz, "User mobility pattern scheme for location update and paging in wireless systems," IEEE Trans. Mobile Comput. July 2002.

[6] Zuji Mao,Christos Douligeris "Group Registration for Reducing Signaling Traffic in Location Tracking" 2010.

[7] Michael Starsinic "System Architecture Challenges in the Home M2M Network" 2010.

[8] Tracy Tung and Abbas Jamalipour "Adaptive Location Management Strategy Combining Distance-Based Update and Sectional Paging Techniques" 2003.

[9] Gregory P. Pollini, Member, IEEE, and Chih-Lin I, Member, IEEE"A Profile-Based Location Stategy and Its Performance" IEEE JOURNAL ON SELECTED AREAS IN COMMUNICATIONS, VOL. 15, NO. 8, OCTOBER 1997.

[10] Rung-Hung Gau, and Ching-Pei Cheng, Member, IEEE "Optimal Tree Pruning for Location Update in Machine-to-Machine Communications" IEEE 


\section{TRANSACTIONS ON WIRELESS}

COMMUNICATIONS, VOL. 12, NO. 6, JUNE.2013.

[11] Ali Khosrozadeh, Sanaz Pashmforoush, Abolfazl Akbari, Maryam Bagheri, Neda Beikmahdavi "Presenting a Novel Page Replacement Algorithm Based on LRU" J. Basic. 2012.

[12] Erdal Cayirci, Member, IEEE, and Ian F. Akyildiz, Fellow, IEEE "Optimal Location Area Designto Minimize Registration Signaling Traffic in Wireless Systems" IEEE TRANSACTIONS ON MOBILE COMPUTING, VOL. 2, NO. 1, JANUARY-MARCH 2003.

[13] A. Bar-Noy and I. Kessler, "Tracking mobile users in wireless communication networks," IEEE Trans. Inf. Theory, vol. 39, no. 6, pp. 1877-1886, Nov. 1993.

[14] I. F. Akyildiz, J. Mcnair, J. S. M. Ho, H. Uzunalioglu, and W. Wang, "Mobility management in next generation wireless systems," Proceedings of the IEEE, Vol. 87, No. 8, Aug. 1999, pp. 1347-1384.

[15] Z. Mao and C. Douligeris, "A location-based mobility tracking scheme for PCS networks," Computer Comm. vol. 23, no. 18, Dec. 2000, pp. 1729-1739.

[16] V. Wong and V. Leung, "An adaptive distance-based location location update algorithm for next-generation PCS networks" IEEE J. Areas Commun., vol. 19, no. 10, Oct. 2001, pp,1952. 